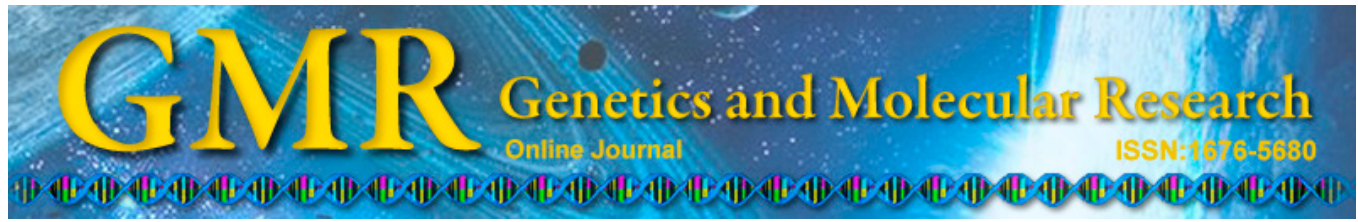

\title{
Meta-analysis of the association between the HNF1B rs4430796 (A>G) polymorphism and risk of prostate cancer based on case-control studies
}

\author{
Y. Zhao*, J. Liang*, J.G. Qi, N. Yang, G. Wu, Y.L. Lin, J.Y. Cao, \\ Q. Wang and Q.C. Wang \\ Department of Urology, Xuzhou Cancer Hospital, \\ Affiliated Hospital of Jiangsu University, Xuzhou, China \\ *These authors contributed equally to this study. \\ Corresponding author: J.G. Qi \\ E-mail:601127797@qq.com
}

Genet. Mol. Res. 14 (3): 7426-7435 (2015)

Received September 4, 2014

Accepted April 6, 2015

Published July 3, 2015

DOI http://dx.doi.org/10.4238/2015.July.3.18

\begin{abstract}
Genome-wide studies have reported an association between the HNF1B rs4430796 $(\mathrm{A}>\mathrm{G})$ polymorphism and prostate cancer risk, but results have been inconsistent and recent meta-analyses have been inadequate. This study aimed to integrate previous results and explore the validity of this association. Electronic searches for all relevant publications through May 18, 2014, were conducted across several databases. Additional studies were identified manually, and only the most recent or complete were used in this meta-analysis. Crude odds ratios (ORs) with 95\% confidence intervals (CIs) were used to assess the strength of the association. Seven eligible case-control studies were identified, incorporating a total of 14,049 patients and 12,674 controls. Overall, we found that the rs4430796 (A>G) polymorphism had a decreased risk of prostate cancer $(\mathrm{GG} v s \mathrm{AA}: \mathrm{OR}=0.661,95 \% \mathrm{CI}=$ $0.615-0.710, \mathrm{P}=0.304 ; \mathrm{AG} v s \mathrm{AA}: \mathrm{OR}=0.782,95 \% \mathrm{CI}=0.739-0.828$, $\mathrm{P}=0.435$; dominant model: $\mathrm{OR}=0.743,95 \% \mathrm{CI}=0.704-0.784, \mathrm{P}=$
\end{abstract}


0.912; recessive model: $\mathrm{OR}=0.764,95 \% \mathrm{CI}=0.718-0.813, \mathrm{P}=0.01$ ). Furthermore, in the stratified analysis, there were significantly decreased risks among studies with population- and hospital-based controls. In the subgroup analysis by ethnicity, significantly decreased risks were also found among Caucasians, Americans, and Asians. Our results suggested that the HNF1B rs4430796 (A>G) polymorphism decreased the risk of prostate cancer. In the future, additional and larger studies on patients from across of the world might be required to validate our findings.

Key words: $H N F 1 B$ rs4430796 (A>G) polymorphism; Prostate cancer; Meta-analysis

\section{INTRODUCTION}

Prostate cancer is a worldwide major public health concern and is the second leading cause of death from cancer in men (Jemal et al., 2009, 2011). In Europe, prostate cancer has the highest incidence apart from skin cancer, and it is the third most common type of cancer after colorectal and lung cancer (Ferlay et al., 2012). It is usually diagnosed at an early stage and many diagnoses are made in asymptomatic men (Lyratzopoulos et al., 2010). Prostate cancer is also a heterogeneous and complex disease caused by interactions of both metabolic and genetic factors. Known metabolic factors include hypertension, hypertriglyceridemia, hypercholesterolemia, and diabetes (Bravi et al., 2006; Bhindi et al., 2014; Ozbek et al., 2014). Furthermore, many inherited genetic variants have been reported to be associated with prostate cancer risk up to date (Na et al., 2013), but few of these candidate-gene associations have been consistently replicated, indicating that the precise molecular mechanisms of prostate cancer are still not entirely clear.

Hepatocyte nuclear factor-1 beta (HNF1B) was shown to be a transcription factor involved in the tissue-specific regulation of embryonic development and gene expression of various organs, such as liver, intestine, kidney, pancreas, and the genitourinary system (Igarashi et al., 2005). The protein consists of a Pit-1/Oct-1/Unc-86 domain, an N-terminal dimerization domain, a homeodomain that mediates DNA binding, and a C-terminal transcriptional activation domain (Hiesberger et al., 2005). In addition, HNF1B is also a member of the homeodomain-containing transcription factor superfamily, which has diverse roles in development and is associated with solid tumors in various forms (Cillo et al., 1999). Published studies suggest that the mRNA expression level of the HNF1B gene might be an important determinant in the development of prostate cancer (Harries et al., 2010).

To date, various single nucleotide polymorphisms (SNPs) have been discovered in the $H N F 1 B$ gene, which is located on 17q21.3 (Tronche and Yaniv, 1992). Several studies have also identified numerous SNPs in the $H N F 1 B$ gene as being associated with the risk of cancers of the ovary and prostate glands (Kao et al., 2012; Chornokur et al., 2013), indicating that genetic variation in $H N F 1 B$ might play an important role in the etiology of numerous cancers. Genomewide association studies have reported the HNF1B rs4430796 (A>G) polymorphism to be associated with a risk of prostate cancer (Gudmundsson et al., 2007; Sun et al., 2008; Liu et al., 2011; Zhou et al., 2011; Zhang et al., 2012; Chan et al., 2013; Rojas et al., 2014).

Although previous studies have focused on the association between rs4430796 (A>G) and prostate cancer susceptibility (Gudmundsson et al., 2007; Sun et al., 2008; Liu et al., 2011; Zhou et al., 2011; Zhang et al., 2012; Chan et al., 2013; Rojas et al., 2014), the results reported 
were from small and highly underpowered studies. In order to resolve these debatable results, a systematic review of published case-control studies was considered useful to better compare results between studies. Therefore, we performed a meta-analysis on all eligible case-control studies, involving a total of 14,049 patients and 12,674 controls, to derive a more precise estimation of the association of rs4430796 (A>G) with susceptibility to prostate cancer. To further confirm the association between rs4430796 and prostate risk in different ethnicities and sources, we conducted additional stratified analyses in this study as well.

\section{MATERIAL AND METHODS}

\section{Identification and eligibility of relevant studies}

To identify all articles that examined the association between HNF1B rs4430796 $(A>G)$ and prostate cancer risk, we searched the electronic literature in PubMed for all relevant articles (the last search update was May 18, 2014, using the following search terms: "HNF1B" or "rs4430796", "genetic variant" or "polymorphism", "prostate cancer" or "tumor of prostate"). Additional studies were identified by a hand search of the references of original studies. The search was limited to English-language articles. Articles included in the meta-analysis were performed with human subjects, and were published in primary literature. The retrieved reports were reviewed to assess their appropriateness for the inclusion in this meta-analysis. Case reports, conference abstracts, review articles, and letters were excluded. When more than one study of the same population was included in several publications, only the most recent or most complete study was used. As a result, seven eligible case-control studies were included.

\section{Data extraction and assessment of study quality}

Data were extracted and entered into a database. Two investigators (Y.Z. and G.W.) independently extracted the information from all eligible publications. Discrepancies were adjudicated by a third reviewer (J.G.Q.) until consensus was achieved on every item. The following information was extracted from each study: surname of the first author, the year of publication, country of origin, ethnicity, source of control groups (population-, or hospital-based controls), genotyping method, and number of patients and controls. For studies including subjects of different ethnic groups, data were extracted separately for each ethnic group when it was possible. Different ethnicities were categorized as Caucasian, American, and Asian. We also assessed the homogeneity of the study population.

\section{Meta-analysis}

The risks (odds ratios, OR) of prostate cancer associated with the HNF1B rs4430796 $(A>G)$ polymorphism were estimated for each publication independently. The risks for $\mathrm{G} / \mathrm{G}$ versus $A / A, A / G$ versus $A / A, A / G$ plus $G / G$ versus $A / A(d o m i n a n t)$, and $G / G$ versus $A / G$ plus $\mathrm{A} / \mathrm{A}($ recessive $)$ were estimated.

\section{Statistical analysis}

The strength of the association between the HNF1B rs4430796 (A>G) polymorphism 
and prostate cancer risk was estimated by ORs with $95 \%$ confidence intervals (CIs). A statistical test for heterogeneity was performed based on the Q statistic (Handoll, 2006), in consideration of the possibility of heterogeneity across the studies. If the $\mathrm{P}$ value of the $\mathrm{Q}$ test, which indicates a lack of heterogeneity among studies, was $>0.05$, the summary OR estimate of each study was calculated by the fixed-effect model (the Mantel-Haenszel method) (Mantel and Haenszel, 1959). Otherwise, the random-effect model (the DerSimonian and Laird method) was used (DerSimonian and Laird, 1986). Stratified analyses were also performed by ethnicity and source of controls. Sensitivity analyses were performed to assess the stability of the results; specifically, a single study in the meta-analysis was deleted in each permutation to reflect the influence of the individual data set to the pooled ORs. In addition, both the funnel plot and the Egger test were used to assess the publication bias (Egger et al., 1997). The significance of asymmetry was determined by $t$ test, and $\mathrm{P}<0.01$ was considered a significant publication bias. Hardy-Weinberg equilibrium (HWE) was tested by the chi-square test. All statistical analyses were performed in Statistical Analysis System software (version 11.0; StataCorp LP, College Station, TX, USA), using two-sided P values.

\section{RESULTS}

\section{Characteristics of studies}

Studies focusing on the $H N F 1 B$ rs4430796 (A>G) polymorphism and prostate cancer were chosen. A total of 26 studies were identified after excluding irrelevant articles. However, after obtaining and reading the full articles, 19 of these were excluded: 3 studies were excluded because they were review articles, 5 because they only included cases, and 11 because of no data of interest or have no raw data. Finally, a total of 7 eligible studies involving 14,049 patients and 12,674 controls were included in the pooled analyses (Gudmundsson et al., 2007; Sun et al., 2008; Liu et al., 2011; Zhou et al., 2011; Zhang et al., 2012; Chan et al., 2013; Rojas et al., 2014). The specific process of eligible study inclusion and exclusion is shown in Figure 1.

The characteristics of the studies selected are summarized in Table 1. These eligible publications included populations from Iceland, The Netherlands, Spain, The USA, Sweden, Finland, France, Japan, China, Singapore, and Chile. All studies were case-control studies. There were 7 studies of Caucasians, 5 of Americans, and 4 of Asians. Prostate cancers were confirmed histologically or pathologically in most studies. Furthermore, controls were primarily matched for age and gender in most studies, ten of which were population-based and six were hospitalbased. Genotype distributions among the controls of all studies were in agreement with HWE.

\section{Overall analysis of data and subgroup analyses}

The main results of this meta-analysis and of the heterogeneity tests are summarized in Table 2 and Figure 2. Overall, the results of this meta-analysis showed that there was a statistically significant association between the $H N F 1 B$ rs4430796 (A>G) polymorphism and a decreased risk of prostate cancer for each genetic model $[\mathrm{OR}=0.661,95 \% \mathrm{CI}=0.615-0.710$, $\mathrm{P}=0.304$ for $\mathrm{GG}$ versus $\mathrm{AA} ; \mathrm{OR}=0.782,95 \% \mathrm{CI}=0.739-0.828, \mathrm{P}=0.435$ for $\mathrm{AG}$ versus $\mathrm{AA}$; $\mathrm{OR}=0.743,95 \% \mathrm{CI}=0.704-0.784, \mathrm{P}=0.912$ for $\mathrm{GG} / \mathrm{AG}$ versus $\mathrm{AA}$ (dominant); $\mathrm{OR}=0.764$, $95 \% \mathrm{CI}=0.718-0.813, \mathrm{P}=0.01$ for $\mathrm{GG}$ versus $\mathrm{AG} / \mathrm{AA}($ recessive) $]$. Additionally, subgroup analyses were conducted to address the effects of different sources of controls and ethnicities. 


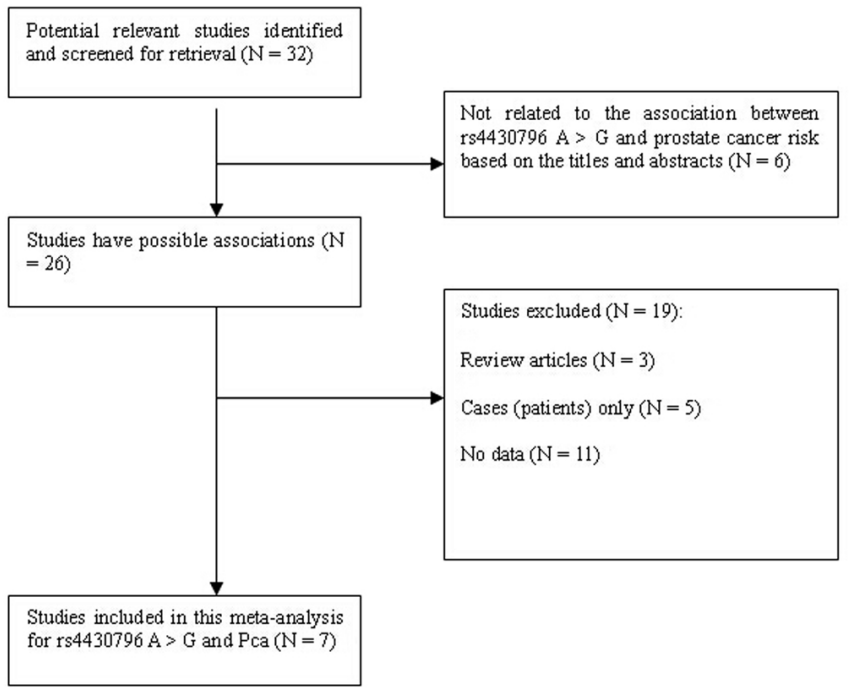

Figure 1. Flow diagram of included and excluded studies. $\mathrm{PCa}=$ prostate cancer.

\begin{tabular}{|c|c|c|c|c|c|c|c|c|c|c|c|c|c|}
\hline \multirow[t]{2}{*}{ Author } & \multirow[t]{2}{*}{ Country } & \multirow[t]{2}{*}{ Ethnicity } & \multirow{2}{*}{$\begin{array}{l}\text { Patients } \\
\text { (N) }\end{array}$} & \multirow{2}{*}{$\begin{array}{l}\text { Controls } \\
\text { (N) }\end{array}$} & \multirow{2}{*}{ Source of controls } & \multirow[t]{2}{*}{ Genotyping method } & \multicolumn{3}{|c|}{ Patients } & \multicolumn{3}{|c|}{ Controls } & \multirow[t]{2}{*}{ HWE } \\
\hline & & & & & & & $\mathrm{A} / \mathrm{A}$ & $\mathrm{A} / \mathrm{G}$ & $\mathrm{G} / \mathrm{G}$ & $\mathrm{A} / \mathrm{A}$ & $\mathrm{A} / \mathrm{G}$ & $\mathrm{G} / \mathrm{G}$ & \\
\hline \multirow{4}{*}{$\begin{array}{l}\text { Gudmundsson } \\
\text { et al. (2007) }\end{array}$} & Iceland & Caucasian & 1474 & 1860 & Population-based & Illumina Hap300 SNP chip & 467 & 709 & 298 & 466 & 930 & 464 & 0.999 \\
\hline & Netherlands & Caucasian & 983 & 1442 & Population-based & Illumina Hap300 SNP chip & 305 & 502 & 176 & 387 & 688 & 367 & 0.083 \\
\hline & Spain & Caucasian & 451 & 1073 & Hospital-based & Illumina Hap300 SNP chip & 101 & 220 & 130 & 209 & 556 & 308 & 0.138 \\
\hline & USA & American & 531 & 500 & Population-based & Illumina Hap300 SNP chip & 156 & 285 & 90 & 127 & 222 & 151 & 0.014 \\
\hline \multirow{7}{*}{ Sun et al. (2008) } & Sweden & Caucasian & 2874 & 1078 & Population-based & PCR-RFLP & 1073 & 1355 & 446 & 509 & 883 & 316 & 0.050 \\
\hline & USA & American & 1521 & 479 & Hospital-based & PCR-RFLP & 488 & 779 & 254 & 120 & 253 & 106 & 0.210 \\
\hline & Finland & Caucasian & 901 & 902 & Population-based & TaqMan & 419 & 395 & 87 & 335 & 431 & 136 & 0.891 \\
\hline & France & Caucasian & 620 & 618 & Population-based & TaqMan & 163 & 308 & 149 & 148 & 309 & 161 & 0.991 \\
\hline & USA & American & 581 & 591 & Population-based & iSelect Infinium assay & 179 & 289 & 113 & 138 & 300 & 153 & 0.699 \\
\hline & Mixed-country & Caucasian & 1121 & 1048 & Population-based & Illumina Hap300 SNP chip & 345 & 522 & 254 & 262 & 529 & 257 & 0.757 \\
\hline & USA & American & 1716 & 1718 & Population-based & TaqMan & 516 & 843 & 357 & 434 & 850 & 434 & 0.664 \\
\hline Liu et al. (2011) & Japan & Asian & 521 & 323 & Hospital-based & TaqMan & 252 & 214 & 55 & 129 & 149 & 45 & 0.851 \\
\hline Zhou et al. (2011) & China & Asian & 105 & 78 & Hospaital-based & PCR-HRM & 59 & 34 & 12 & 38 & 34 & 6 & 0.670 \\
\hline Zhang et al. (2012) & China & Asian & 195 & 160 & Hospital-based & TaqMan & 119 & 60 & 16 & 77 & 73 & 10 & 0.178 \\
\hline Chan et al. (2013) & Singapore & Asian & 289 & 141 & Population-based & Mixed & 169 & 99 & 21 & 67 & 63 & 11 & 0.469 \\
\hline Rojas et al. (2014) & Chile & American & 166 & 33 & Hospital-based & TaqMan & 80 & 75 & 11 & 14 & 15 & 4 & 0.995 \\
\hline
\end{tabular}

HWE $=$ Hardy-Weinberg equilibrium; SNP = single nucleotide polymorphism; PCR-RFLP = polymerase chain reaction-restriction fragment length polymorphism; PCR-HRM = PCR-high resolution melting.

The results indicated that the $H N F 1 B$ rs4430796 (A>G) polymorphism significantly decreased prostate cancer risk among studies with population-based controls $(\mathrm{OR}=0.651,95 \% \mathrm{CI}=$ $0.602-0.704, \mathrm{P}=0.302$ for $\mathrm{GG}$ versus $\mathrm{AA} ; \mathrm{OR}=0.791,95 \% \mathrm{CI}=0.743-0.842, \mathrm{P}=0.246$ for $\mathrm{AG}$ versus $\mathrm{AA} ; \mathrm{OR}=0.746,95 \% \mathrm{CI}=0.703-0.791, \mathrm{P}=0.731$ for $\mathrm{GG}+\mathrm{AG}$ versus $\mathrm{AA} ; \mathrm{OR}=$ $0.748,95 \% \mathrm{CI}=0.700-0.800, \mathrm{P}=0.012$ for $\mathrm{GG}$ versus $\mathrm{AG}+\mathrm{AA}$ ) as well as hospital-based controls $(\mathrm{OR}=0.719,95 \% \mathrm{CI}=0.598-0.866, \mathrm{P}=0.328$ for $\mathrm{GG}$ versus $\mathrm{AA} ; \mathrm{OR}=0.737,95 \% \mathrm{CI}=$ $0.639-0.849, \mathrm{P}=0.702$ for $\mathrm{AG}$ versus $\mathrm{AA} ; \mathrm{OR}=0.729,95 \% \mathrm{CI}=0.638-0.834, \mathrm{P}=0.833$ for GG+AG versus $A A)$. In the subgroup analysis by ethnicity, statistically significant decreased risks were found among Caucasians $(\mathrm{OR}=0.676,95 \% \mathrm{CI}=0.618-0.739, \mathrm{P}=0.15$ for $\mathrm{GG}$ ver- 
sus $\mathrm{AA} ; \mathrm{OR}=0.780,95 \% \mathrm{CI}=0.727-0.838, \mathrm{P}=0.419$ for $\mathrm{AG}$ versus $\mathrm{AA} ; \mathrm{OR}=0.746,95 \% \mathrm{CI}$ $=0.698-0.798, \mathrm{P}=0.549$ for $\mathrm{GG}+\mathrm{AG}$ versus $\mathrm{AA} ; \mathrm{OR}=0.791,95 \% \mathrm{CI}=0.733-0.854, \mathrm{P}=$ 0.025 for $\mathrm{GG}$ versus $\mathrm{AG}+\mathrm{AA})$, Americans $(\mathrm{OR}=0.616,95 \% \mathrm{CI}=0.539-0.703, \mathrm{P}=0.453$ for $\mathrm{GG}$ versus $\mathrm{AA} ; \mathrm{OR}=0.829,95 \% \mathrm{CI}=0.743-0.926, \mathrm{P}=0.467$ for $\mathrm{AG}$ versus $\mathrm{AA} ; \mathrm{OR}=0.757$, $95 \% \mathrm{CI}=0.682-0.840, \mathrm{P}=0.831$ for $\mathrm{GG}+\mathrm{AG}$ versus $\mathrm{AA} ; \mathrm{OR}=0.694,95 \% \mathrm{CI}=0.621-0.800$, $\mathrm{P}=0.069$ for $\mathrm{GG}$ versus $\mathrm{AG}+\mathrm{AA})$, and Asians $(\mathrm{OR}=0.653,95 \% \mathrm{CI}=0.534-0.800, \mathrm{P}=0.689$ for $\mathrm{AG}$ versus $\mathrm{AA}$; $\mathrm{OR}=0.672,95 \% \mathrm{CI}=0.555-0.814, \mathrm{P}=0.888$ for $\mathrm{GG}+\mathrm{AG}$ versus $\mathrm{AA}$ ).

Table 2. Meta-analysis of the $H N F 1 B$ rs $4430796 \mathrm{~A}>\mathrm{G}$ polymorphism and risk of prostate cancer.

\begin{tabular}{|c|c|c|c|c|c|c|c|c|c|}
\hline \multirow[t]{2}{*}{ Variables } & \multirow[t]{2}{*}{$\mathrm{N}^{\mathrm{a}}$} & \multicolumn{2}{|l|}{ GG $v s$ AA } & \multicolumn{2}{|l|}{ AG vs AA } & \multicolumn{2}{|c|}{ GG+AG vs AA (dominant) } & \multicolumn{2}{|c|}{ GG vs AG+AA (recessive) } \\
\hline & & OR $(95 \% \mathrm{CI})$ & $\mathrm{P}$ & OR $(95 \% \mathrm{CI})$ & $\mathrm{P}$ & OR $(95 \% \mathrm{CI})$ & $\mathrm{P}$ & OR $(95 \% \mathrm{CI})$ & $\mathrm{P}^{\mathrm{b}}$ \\
\hline Total & 16 & $0.661(0.615-0.710)$ & 0.304 & $0.782(0.739-0.828)$ & 0.435 & $0.743(0.704-0.784)$ & 0.912 & $0.764(0.718-0.913)$ & 0.01 \\
\hline \multicolumn{10}{|l|}{ Ethnicities } \\
\hline Caucasian & 7 & $0.676(0.618-0.739)$ & 0.15 & $0.780(0.727-0.838)$ & 0.419 & $0.746(0.698-0.798)$ & 0.549 & $0.791(0.733-0.854)$ & 0.025 \\
\hline American & 5 & $0.616(0.539-0.703)$ & 0.453 & $0.829(0.743-0.926)$ & 0.467 & $0.757(0.682-0.840)$ & 0.831 & $0.694(0.621-0.776)$ & 0.069 \\
\hline Asian & 4 & $0.758(0.543-1.057)$ & 0.533 & $0.653(0.534-0.800)$ & 0.689 & $0.672(0.555-0.814)$ & 0.888 & $0.903(0.658-1.241)$ & 0.4 \\
\hline \multicolumn{10}{|l|}{ Source of controls } \\
\hline Population-based & 10 & $0.651(0.602-0.704)$ & 0.302 & $0.791(0.743-0.842)$ & 0.246 & $0.746(0.703-0.791)$ & 0.731 & $0.748(0.700-0.800)$ & 0.012 \\
\hline Hospital-based & 6 & $0.719(0.598-0.866)$ & 0.328 & $0.737(0.639-0.849)$ & 0.702 & $0.729(0.638-0.834)$ & 0.833 & $0.856(0.732-1.001)$ & 0.181 \\
\hline
\end{tabular}

${ }^{\mathrm{a}}$ Number of comparisons; ${ }^{\mathrm{b}} \mathrm{P}$ value of Q-test for heterogeneity test. $\mathrm{OR}=$ odds ratio; $\mathrm{CI}=$ confidence interval.

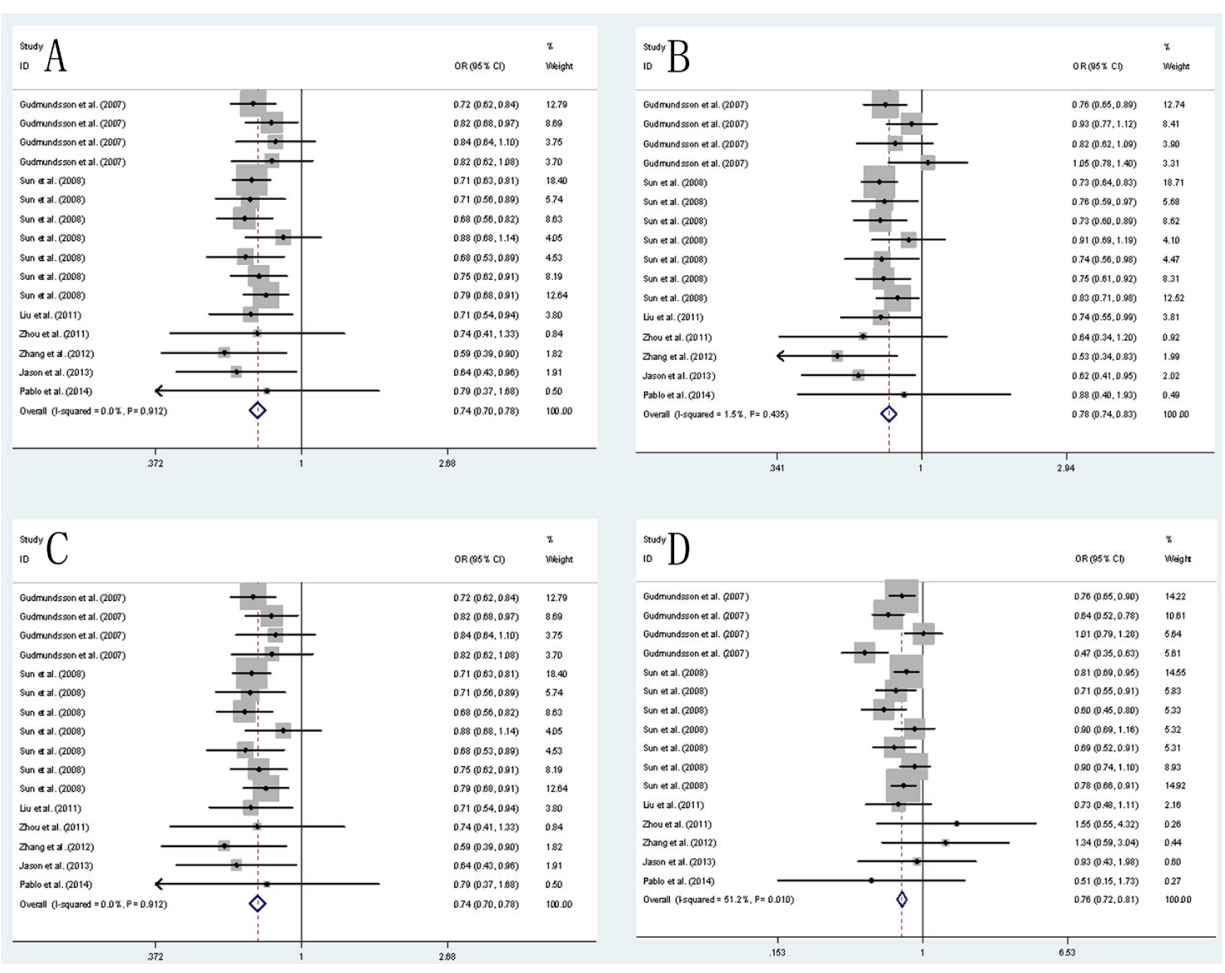

Figure 2. Forest plots for the overall association between the $N H F 1 B$ rs4430796 (A $>\mathrm{G})$ polymorphism and prostate cancer risk. $\mathrm{OR}$, odds ratio; $\mathrm{CI}$, confidence interval. $\mathrm{A} . \mathrm{OR}=0.661,95 \% \mathrm{CI}=0.615-0.710, \mathrm{P}=0.304$ for $\mathrm{GG}$ versus $\mathrm{AA}$; B. $\mathrm{OR}=0.782,95 \% \mathrm{CI}=0.739-0.828, \mathrm{P}=0.435$ for $\mathrm{AG}$ versus $\mathrm{AA} ; \mathrm{C} . \mathrm{OR}=0.743,95 \% \mathrm{CI}=0.704-0.784, \mathrm{P}=0.912$ for $\mathrm{GG} / \mathrm{AG}$ versus $\mathrm{AA}$ (dominant); $\mathbf{D}$. $\mathrm{OR}=0.764,95 \% \mathrm{CI}=0.718-0.813, \mathrm{P}=0.01$ for $\mathrm{GG}$ versus AG/AA (recessive). 


\section{Sensitivity analysis}

As shown in Figure 3, meta-analyses were conducted sequentially following removal of each particular study. The results showed that fixed-effect and/or random-effect estimates before and after the deletion of each study were similar overall, suggesting that the results of this meta-analysis are stable.

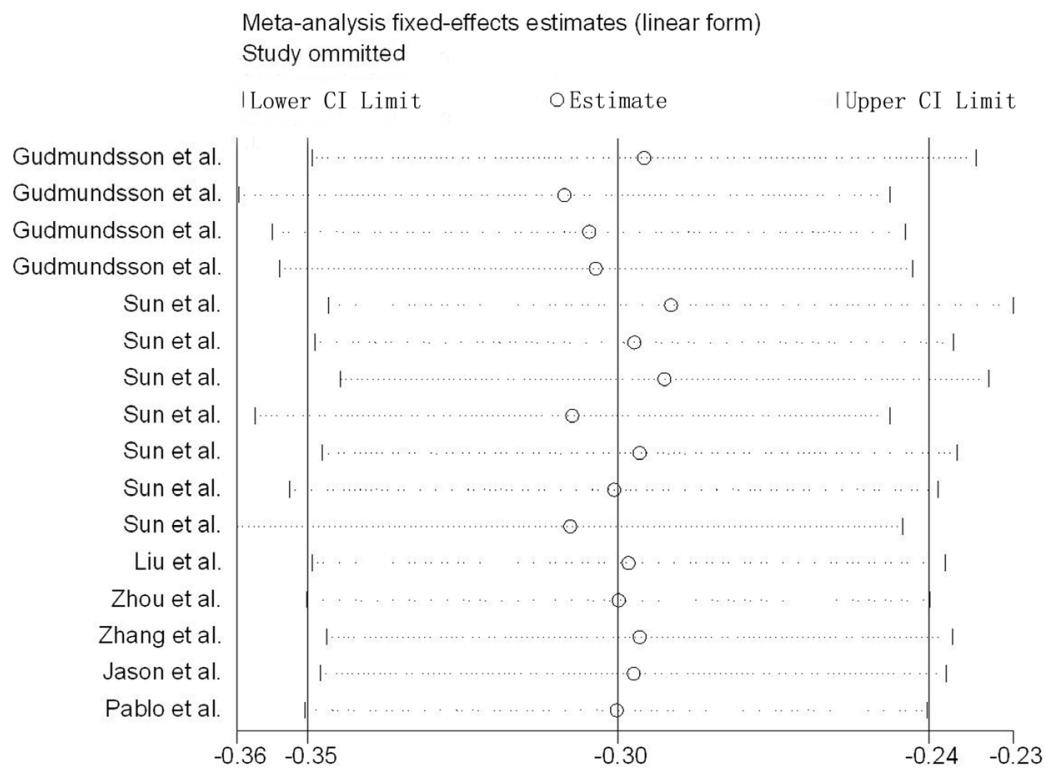

Figure 3. Influence analysis of the summary odds ratio coefficients on the association of the rs $4430796 \mathrm{~A}>\mathrm{G}$ SNP with prostate cancer risk (the combined group of GG+GA genotypes was compared with the AA genotype). Results were computed by omitting each study (on the bottom) in turn. Bars, 95\% confidence interval. Meta-analysis random-effect estimates (linear form) were used.

\section{Publication bias}

Funnel plots and the Egger tests were performed to assess the publication bias. The shapes of the funnel plots did not reveal any evidence of obvious asymmetry under any compared models. The Egger's test was used to provide statistical evidence of funnel plot symmetry. In agreement with the original assessment, the Egger test results also did not show any evidence of publication bias ( $\mathrm{P}>0.05$ for $\mathrm{GG}+\mathrm{AG}$ versus AA; Figure 4).

\section{DISCUSSION}

The present meta-analysis, including 14,049 patients and 12,674 controls from seven case-control studies, explored the association between the HNF1B rs4430796 (A>G) polymorphism and prostate cancer risk. To our knowledge, this is the most comprehensive metaanalysis to examine this association. Our results showed that the rs4430796 (A>G) polymorphism was associated with a decreased risk of prostate cancer. Given the important roles of 


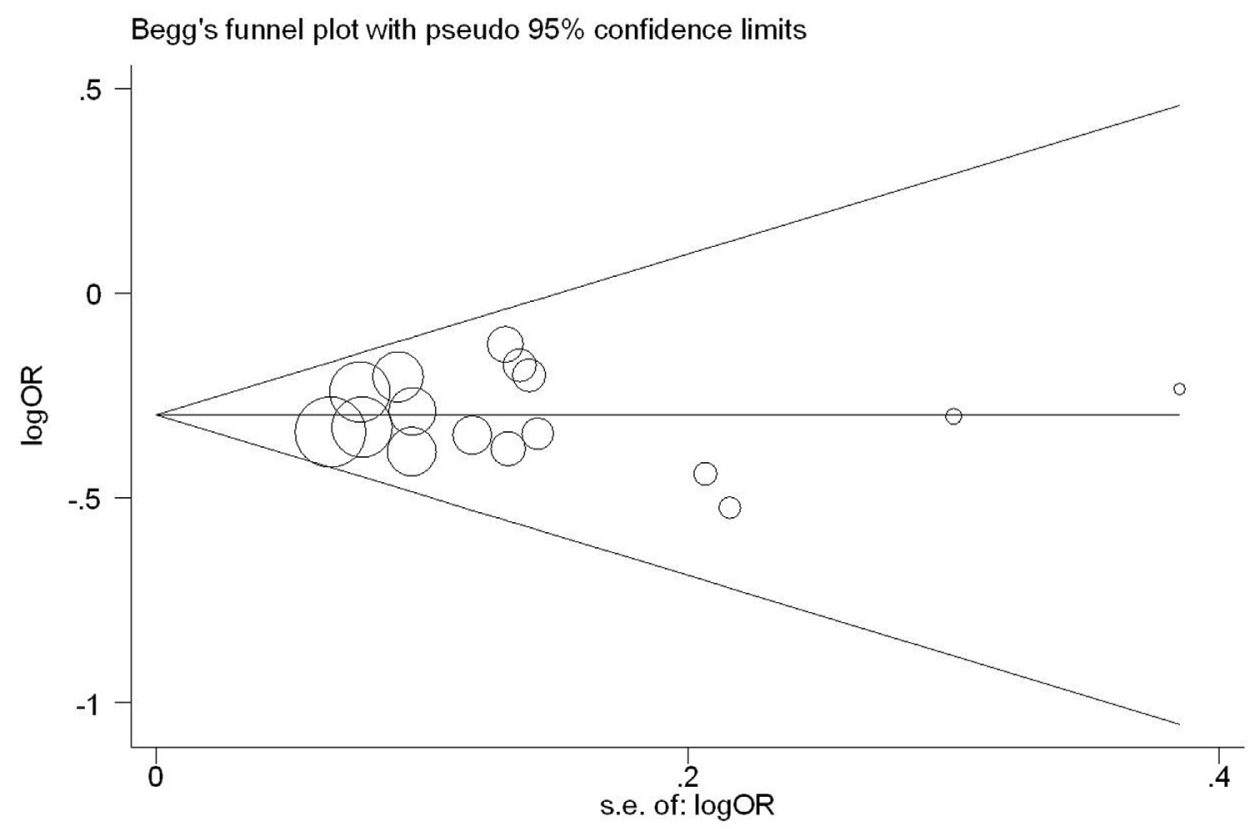

Figure 4. Begg's funnel plot with pseudo $95 \%$ confidence limits of publication bias for the $H N F 1 B$ rs $4430796 \mathrm{~A}>\mathrm{G}$ polymorphism (GG+AG versus AA). Each point represents a separate study for the indicated association. $\log [\mathrm{OR}]$, natural logarithm of the odds ratio; horizontal line, mean effect size; s.e., standard error.

HNF1B in the regulation of cell proliferation, it is biologically plausible that genetic variation at the $H N F 1 B$ rs 4430796 polymorphic site might modulate the risk of prostate cancer.

Cancer is a multifactorial disease that results from complex interactions between various inherited and environmental factors (Pharoah et al., 2004). This is particularly true for the sporadic forms of cancer that tend to be common in the population, as opposed to familial cancer syndromes. Genetic variations can modify DNA repair capacities and alter cancer risk. To date, approximately 150 human DNA repair genes have been identified. But these known genes account for only a small proportion of the risk of cancer (Wood et al., 2005).

The HNF1B gene, located on 17q21.3, encodes a transcription factor involved in the tissue-specific regulation of gene expression and embryonic development of numerous organs (Tronche and Yoniv, 1992; Igarashi et al., 2005). In addition, HNF1B binds to promoters of target genes as heterodimers or homodimers and can either activate or repress transcription (Hiesberger et al., 2005). To date, dysregulation of HNF1B has been detected within various forms of solid tumors (Cillo et al., 1999). Over the past decades, HNF1B genetic variation has received widespread attention, and $\mathrm{rs} 4430796(\mathrm{~A}>\mathrm{G})$ has become the most studied SNP in $H N F 1 B$. Recently, many studies have been conducted to investigate the associations between this polymorphism and prostate cancer risk across different countries. Most have reported a role for rs4430796 in prostate cancer risk (Gudmundsson et al., 2007; Sun et al., 2008; Liu et al., 2011; Zhang et al., 2012; Chan et al., 2013). However, Zhou et al. (2011) and Rojas et al. (2014) found that there was no significant association between the rs4430796 (A>G) polymorphism and the risk of prostate cancer (dominant model). This could be explained 
in part by the possible small effect of the polymorphism on prostate cancer risk and by the relatively small sample size in each published report. To derive a more precise estimation, a meta-analysis from seven case-control studies was performed. The results indicated that the $H N F 1 B$ rs4430796 $(\mathrm{A}>\mathrm{G})$ polymorphism significantly decreased the risk of prostate cancer in the populations studied overall.

Additionally, in the analyses stratified by population- or hospital-based controls, significant associations were also detected, indicating that the different sources of controls did not influence the association. The same result was also found in the analysis stratified by ethnicity, with significant associations detected in Caucasians, Americans, and Asians, suggesting that ethnic differences in genetic backgrounds and the environmental/life style context did not play an obvious role in the HNF1B rs4430796 (A>G) polymorphism association with prostate cancer risk.

Several highlights merited adequate consideration, which distinguished the present investigation from those previously published. First, this was the largest synthesis exploring the relationship of the $H N F 1 B$ rs4430796 (A>G) polymorphism with the risk of prostate cancer, and it derived the most precise estimation available to date. Second, our results were credible and stabilized because of the low probability of publication bias. Third, most of the results from the present study were in accordance with those of the corresponding meta-analysis, which might be a reflection of the credibility of the results.

In addition, limitations of this meta-analysis should be acknowledged. First, the overall outcomes were based on individual unadjusted ORs, while a more precise evaluation should be adjusted by other potentially suspected factors (i.e., age, smoking, and environmental factors) if such information is widely available. Second, most meta-analyses encountered difficulties with unpublished studies. Owing to only published studies having been included in our meta-analysis, it was likely that some unpublished studies might have been missed. Therefore, we might miss a chance to obtain a relatively larger sample size and increased statistical power. Third, due to lack of uniformity in the controls and the populations, which came from different countries, certain results should be interpreted carefully.

In summary, based on a larger sample size than previously utilized, this meta-analysis indicated that the HNF1B rs4430796 (A>G) polymorphism decreased the risk of prostate cancer based on current published data. Thus, the HNF1B rs4430796 (A>G) polymorphism might be an independent protective factor for prostate cancer. Further investigation with more detailed individual data including a wider spectrum of subjects should be carried out to investigate the association between $H N F 1 B$ polymorphisms and the risk of prostate cancer in combination with other potential prostate cancer risks.

\section{ACKNOWLEDGMENTS}

Research not supported by any financial. The authors were fully responsible for all content and editorial decisions.

\section{REFERENCES}

Bhindi B, Locke J, Alibhai SM, Kulkarni GS, et al. (2014). Dissecting the association between metabolic syndrome and prostate cancer risk: analysis of a large clinical cohort. Eur. Urol. 67: 64-70.

Bravi F, Scotti L, Bosetti C, Talamini R, et al. (2006). Self-reported history of hypercholesterolaemia and gallstones and 
the risk of prostate cancer. Ann. Oncol. 17: 1014-1017.

Chan JY, Li H, Singh O, Mahajan A, et al. (2013). 8q24 and 17q prostate cancer susceptibility loci in a multiethnic Asian cohort. Urol. Oncol. 31: 1553-1560.

Chornokur G, Amankwah EK, Davis SN, Phelan CM, et al. (2013). Variation in HNF1B and obesity may influence prostate cancer risk in African American men: a pilot study. Prostate Cancer 2013: 384594.

Cillo C, Faiella A, Cantile M and Boncinelli E (1999). Homeobox genes and cancer. Exp. Cell Res. 248: 1-9.

DerSimonian R and Laird N (1986). Meta-analysis in clinical trials. Control. Clin. Trials 7: 177-188.

Egger M, Davey Smith G, Schneider M and Minder C (1997). Bias in meta-analysis detected by a simple, graphical test. BMJ 315: 629-634.

Ferlay J, Steliarova-Foucher E, Lortet-Tieulent J, Rosso S, et al. (2012). Cancer incidence and mortality patterns in Europe: estimates for 40 countries in 2012. Eur. J. Cancer 49: 1374-1403.

Gudmundsson J, Sulem P, Steinthorsdottir V, Bergthorrson JT, et al. (2007). Two variants on chromosome 17 confer prostate cancer risk, and the one in TCF2 protects against type 2 diabetes. Nat. Genet. 39: 977-983.

Handoll HH (2006). Systematic reviews on rehabilitation interventions. Arch. Phys. Med. Rehabil. 87: 875.

Harries LW, Perry JR, McCullagh P and Crundwell M (2010). Alterations in LMTK2, MSMB and HNF1B gene expression are associated with the development of prostate cancer. BMC Cancer 10: 315.

Hiesberger T, Shao X, Gourley E, Reimann A, et al. (2005). Role of the hepatocyte nuclear factor-1beta (HNF-1beta) C-terminal domain in Pkhd1 (ARPKD) gene transcription and renal cystogenesis. J. Biol. Chem. 280: 10578-10586.

Igarashi P, Shao X, McNally BT and Heisberger T (2005). Roles of HNF-1beta in kidney development and congenital cystic diseases. Kidney Int. 68: 1944-1947.

Jemal A, Siegel R, Ward E, Hao Y, et al. (2009). Cancer statistics. CA Cancer J. Clin. 59: 225-249.

Jemal A, Bray F, Center MM, Ferlay J, et al. (2011). Global cancer statistics. CA Cancer J. Clin. 61: 69-90.

Kao YC, Lin MC, Lin WC, Jeng YM, et al. (2012). Utility of hepatocyte nuclear factor-1 $\beta$ as a diagnostic marker in ovarian carcinomas with clear cells. Histopathology 61: 760-768.

Liu M, Suzuki M, Arai T, Sawabe M, et al. (2011). A replication study examining three common single-nucleotide polymorphisms and the risk of prostate cancer in a Japanese population. Prostate 71: 1023-1032.

Lyratzopoulos G, Barbiere JM, Greenberg DC, Wright KA, et al. (2010). Population based time trends and socioeconomic variation in use of radiotherapy and radical surgery for prostate cancer in a UK region: continuous survey. BMJ 340: c1928.

Mantel N and Haenszel W (1959). Statistical aspects of the analysis of data from retrospective studies of disease. J. Natl. Cancer Inst. 22: 719-748.

Na R, Liu F, Zhang P, Ye D, et al. (2013). Evaluation of reported prostate cancer risk-associated SNPs from genome-wide association studies of various racial populations in Chinese men. Prostate 73: 1623-1635.

Ozbek E, Otunctemur A, Dursun M, Sahin S, et al. (2014). Diabetes mellitus and HbA1c levels associated with high grade prostate cancer. Asian Pac. J. Cancer Prev. 15: 2555-2558.

Pharoah PD, Dunning AM, Ponder BA and Easton DF (2004). Association studies for finding cancer-susceptibility genetic variants. Nat. Rev. Cancer 4: 850-860.

Rojas PA, Torres-Estay V, Cerda-Infante J, Montecinos VP, et al. (2014). Association of a single-nucleotide polymorphism from chromosome 17q12 with the aggressiveness of prostate cancer in a Hispanic population. J. Cancer Res. Clin. Oncol. 140: 783-788.

Sun J, Zheng SL, Wiklund F, Isaacs SD, et al. (2008). Evidence for two independent prostate cancer risk-associated loci in the HNF1B gene at 17q12. Nat. Genet. 40: 1153-1155.

Tronche F and Yaniv M (1992). HNF1, a homeoprotein member of the hepatic transcription regulatory network. Bioessays 14: 579-587.

Wood RD, Mitchell M and Lindahl T (2005). Human DNA repair genes, 2005. Mutat. Res. 577: 275-283.

Zhang YR, Xu Y, Yang K, Liu M, et al. (2012). Association of six susceptibility loci with prostate cancer in northern Chinese men. Asian Pac. J. Cancer Prev. 13: 6273-6276.

Zhou CH, Wang JY, Cao SY, Shi XH, et al. (2011). Association between single nucleotide polymorphisms on chromosome $17 \mathrm{q}$ and the risk of prostate cancer in a Chinese population. Chin. J. Cancer 30: 721-730. 